\title{
Niños españoles con sintomatología depresiva: un estudio de sus quejas somáticas
}

\section{Spanish children with depressive symptoms: a study of their somatic complaints}

\author{
Mireia Orgilés, Iván Fernández-Martínez, Silvia Melero, Alexandra Morales, José P. Espada \\ Universidad Miguel Hernández de Elche, España
}

(Rec: agosto de 2017 - Acept: abril de 2018)

\section{Resumen}

La presencia de síntomas somáticos en población clínica infantil ha sido analizada en estudios internacionales, pero son escasos los trabajos dirigidos a estudiar las características de los síntomas somáticos en niños españoles con sintomatología depresiva. El presente estudio examina la relación entre las quejas somáticas y los síntomas depresivos en una muestra de niños españoles, teniendo en cuenta su sexo y edad. Participaron 666 niños con edades entre 8 y 12 años que completaron el Inventario de Depresión Infantil y el Inventario de Somatización Infantil que examina 35 síntomas somáticos. Los resultados indican que los síntomas pseudoneurológicos, gastrointestinales y de dolor son más frecuentes en los niños con sintomatología depresiva, siendo el dolor de articulaciones, el dolor de cabeza y el cansancio los síntomas más prevalentes. Se concluye la importancia de atender adecuadamente a los síntomas somáticos puesto que con frecuencia pueden responder a un trastorno emocional más que a una enfermedad física.

Palabras Clave: Depresión; quejas somáticas; somatización; población infantil

\begin{abstract}
The presence of somatic symptoms in children has been widely analyzed in international studies, but there is a lack of studies aimed at studying the characteristics of somatic symptoms in Spanish children with depressive symptoms. This study examines the relationship between somatic complaints and depressive symptoms in a sample of Spanish children, taking into account their age and sex. Participants were 666 children aged between 8 and 12 years who completed the Child Depression Inventory and the Children's Somatization Inventory that examine 35 somatic symptoms. The results indicate that pseudoneurological, pain and gastrointestinal symptoms are more common in children with depressive symptoms, being the joint pain, headache and fatigue the most prevalent symptoms. An adequately detection of somatic symptoms in children could be important due to these symptoms often may respond to an emotional disorder rather than a physical illness.
\end{abstract}

Key Words: Depression; somatic complaints; somatization; children 


\section{Introducción}

La depresión infantil es un trastorno frecuente que afecta a entre un 4.2 y un $10 \%$ de niños españoles en edades prepuberales (Bernaras, Jaureguizar, Soroa, Ibabe \& de las Cuevas, 2013; Del Barrio, 2007). Cuando se trata de población clínica su prevalencia asciende al 32\% (López, Alcántara, Fernández, Castro \& López, 2010). Estos datos, a pesar de ser menos elevados que en otros países de habla hispana (Barros-Vanegas, Mercado-Osorio \& Solano-Lamadrid, 2013; Beltrán \& Mora, 2011), ponen de manifiesto que se trata de una problemática clínica necesaria de abordar. Atendiendo a su prevalencia en función del sexo, diferentes investigaciones destacan que para el rango de edad de 8 a 12 años el porcentaje de niños y niñas con sintomatología depresiva es aproximadamente el mismo, siendo ligeramente más elevado en los varones (Bernaras et al., 2013; Del Barrio, 2008). A partir de los 12 años su incidencia aumenta considerablemente en la población infantil femenina (Abad, Forns \& Gómez, 2002; Angold, Erkanli, Silberg, Eaves \& Costello, 2002; Cicchetti \& Cohen, 2006).

Dependiendo de la etapa evolutiva, la manifestación de la depresión varía debido a los cambios biológicos, psicológicos y sociales propios de cada edad. A diferencia de los adultos, la depresión en niños suele caracterizarse por episodios de irritabilidad más que de tristeza, y suele estar asociada a ansiedad, quejas somáticas, alteraciones de sueño y alimentación, entre otros (Guillén, Gordillo, Ruiz, Gordillo \& Gordillo, 2013). Según García-Vera y Sanz (2012), los niños pequeños que sufren depresión suelen mostrar más síntomas de tipo psicofisiológico y motor, mientras que en los niños más mayores son más frecuentes los síntomas cognitivos. La Guía de práctica clínica sobre la depresión mayor en la infancia y en la adolescencia (2009) informa de que en la etapa escolar (entre 7 y 12 años) los síntomas depresivos se manifiestan fundamentalmente a tres niveles. A nivel cognitivo destaca el estado de ánimo disfórico, falta de concentración, disminución del rendimiento escolar, desinterés por el juego, baja autoestima, culpabilidad, frustración, y en ocasiones ideas recurrentes de muerte; a nivel conductual-afectivo los niños suelen mostrarse irritables, agresivos, agitados o por el contrario inhibidos, tienen una sensación constante de aburrimiento, apatía y se aíslan del resto de sus compañeros; y a nivel somático son comunes las cefaleas, dolores abdominales, cansancio, insomnio o hipersomnia, pesadillas recurrentes, alteraciones del apetito y dificultad para alcanzar el peso correspondiente a su edad cronológica. En el DSM-5 se ha incluido el "Trastorno de Estado de Ánimo Disruptivo y No regulado" como una categoría en la que se incluyen aquellos niños que presentan irritabilidad y frecuentes episodios de rabietas tres o más veces a la semana durante más de un año.

Diversos estudios han confirmado que existe una correlación positiva entre la depresión y la presencia de quejas somáticas tanto en población adulta como en población infantil (Campo et al., 2004; Rieffe, Meerum Terwogt \& Jellesma, 2008; Rieffe et al., 2010; Zolog et al., 2011). Además, se ha hallado cierta relación entre la conciencia emocional y las quejas somáticas en niños y adolescentes, actuando los estados de ánimo negativos como mediadores (Kranzler et al., 2016; Rieffe \& De Rooij, 2012). Un reciente estudio llevado a cabo por Ordóñez, Maganto y González (2015) sostiene que la queja somática más común en la población infantil española es el cansancio, seguida del dolor de cabeza y de estómago. En cuanto a las diferencias por sexo, los estudios han demostrado que las niñas tienen mayor predisposición a sufrir síntomas somáticos que los niños, los cuales son más propensos a expresarse a nivel conductual (López et al., 2010; Serra, Claustre \& Bonillo, 2013).

La presencia de síntomas somáticos en población clínica infantil ha sido analizada en estudios internacionales (Anttila et al., 2004; Baji et al., 2009; Shanahan et al., 2015). Sin embargo, son escasos los trabajos que han evidenciado la frecuencia y características de los síntomas somáticos cuando se trata de niños con depresión en nuestro país. Por tanto, el objetivo del presente trabajo es aumentar la literatura científica sobre dicha problemática, examinando la relación entre las quejas somáticas y la sintomatología depresiva en población infantil española, teniendo en cuenta el sexo y la edad de los participantes. Tomando como referencia la literatura científica previa, se hipotetiza que los niños españoles que presentan sintomatología depresiva manifestarán más quejas somáticas que los que no presentan síntomas depresivos, y que las niñas informarán de más quejas somáticas que los niños.

\section{Método}

\section{Participantes}

Participaron en el estudio 666 niños con edades entre 8 y 12 años ( $M=10.02$ años; DT $=1.23$ ), el 50,30\% niñas. El $87 \%$ de los participantes eran españoles y el resto procedían de otros países hispanohablantes. La mayoría de los niños (84\%) vivían con ambos padres, el $15 \%$ tenían padres divorciados, y el resto (1\%) tenían madre o padre soltero o eran huérfanos. El nivel socioeconómico, que se determinó en base al empleo de los padres y a la ubicación del colegio de los niños, era predominantemente medio. Los criterios de inclusión contemplados en este estudio fueron que los participantes tuvieran una edad comprendida entre los 8 y los 12 años de edad, que fueran hispanohablantes y que los padres hubieran firmado y entregado el consentimiento informado.

\section{Instrumentos}

La sintomatología depresiva se evaluó mediante el Inventario de Depresión Infantil (CDl; Kovacs, 1992) dirigido a niños y adolescentes con edades comprendidas entre 7 y 15 años. Está formado por 27 ítems con tres alternativas de respuesta que indican ausencia de sintomatología (0), sintomatología leve (1) y sintomatología depresiva (2). El inventario tiene un rango de puntuaciones de 0 a 54. La versión en español (Del Barrio \& Carrasco, 2004) muestra buenas propiedades psicométricas, con una consistencia interna de .79 y una fiabilidad test-retest de .38 .

El Inventario de Somatización Infantil (CSI; Walker, Beck, Garber y Lambert, 2009) es un autoinforme que consta de 35 ítems que se corresponden con síntomas somáticos frecuentes. Está dirigido a niños de 8 a 12 años que deben valorar la frecuencia con la que han manifestado cada síntoma en las dos últimas semanas en una escala de 5 puntos (de $0=$ nada a 4 = mucho). El inventario, cuyo rango de puntuación oscila de 0 a 96, está formado por tres subescalas que examinan síntomas pseudoneurológicos, síntomas gastrointestinales y síntomas de dolor. La versión española de la prueba de 35 ítems (Orgilés \& Espada, 2014) muestra una consistencia interna de .93. 


\section{Procedimiento}

La muestra se reclutó en ocho colegios públicos y concertados de la provincia de Alicante seleccionados al azar y ubicados en zonas rurales y urbanas, de la costa y del interior, con el objetivo de conseguir la mayor representatividad posible de los centros escolares. Después de solicitar los permisos oportunos a la dirección de los colegios, se obtuvo el consentimiento informado del $95 \%$ de los padres. Las pruebas se completaron en las aulas de forma colectiva. Se distribuyeron los cuestionarios y se informó a los niños de las instrucciones para su cumplimentación, pidiéndoles que levantaran la mano en caso de tener alguna duda. Se les informó de que los resultados eran anónimos y se les pidió que completaran los autoinformes con sinceridad. La Oficina Evaluadora de Proyectos de la universidad de la que forman parte los autores aprobó previamente el estudio.

\section{Análisis de datos}

Se llevó a cabo un Análisis Multivariado de la Varianza (MANOVA), incluyendo el sexo y la presencia o ausencia de sintomatología depresiva como factores fijos, y considerándose como variables dependientes las subescalas del CSI (síntomas pseudoneurológicos, síntomas gastrointestinales y síntomas de dolor). El análisis se ajustó por la edad. Se hallo el tamaño del efecto de Cohen de las diferencias que fueron significativas, considerando 0.20 un tamaño del efecto pequeño, 0.50 medio y 0.80 grande. Se examinaron mediante la prueba $\chi^{2}$ las diferencias entre el porcentaje de niños con sintomatología depresiva y sin sintomatología que presentaban cada queja somática muchas o muchísimas veces (puntuaciones iguales o mayores a 3 en cada ítem). Los análisis estadísticos se reali- zaron con el programa de análisis estadístico SPSS 22.0.

\section{Resultados}

Diferencias en quejas somáticas en función de la sintomatología depresiva y el sexo

Se dicotomizaron las puntuaciones de los participantes en el Inventario de Depresión Infantil, considerándose la puntuación 19 como indicadora de sintomatología depresiva de acuerdo a estudios previos (e.g., Domènech-Llaberia \& Polaino-Lorente, 1990; Ezpeleta, de la Osa, Gratacos \& Pons, 1992). El $8 \%$ de los niños $(n=50)$ presentaron síntomas depresivos y el $92 \%(n=616)$ ausencia de sintomatología. La edad media de ambos grupos fue 10.77 (DT $=1.031$ ) y 10.86 (DT $=0.96$ ) respectivamente, no hallándose diferencias en las características de ambos subgrupos.

Los resultados del MANOVA mostraron diferencias significativas en función del sexo ( $F=5,625 ; p=.001)$ y de la presencia o no de sintomatología depresiva $(F=22,986 ; p=.000)$ en todas las subescalas del CSI $(p<.001)$. Los niños con sintomatología depresiva presentaron más síntomas pseudoneurológicos $(p<.001, \mathrm{~d}=0.46)$, más síntomas gastrointestinales ( $p$ $<.001, d=0.43)$ y más síntomas de dolor $(p<.001, d=0.37)$. Las diferencias fueron significativas en función del sexo en los síntomas pseudoneurológicos y gastrointestinales, mostrando las niñas más quejas de tipo pseudoneurológico $(p<.05 ; \mathrm{d}=$ $0.11)$ y gastrointestinales $(p<.001 ; \mathrm{d}=0.24)$ que los niños. La interacción entre la sintomatología depresiva y el sexo no fue significativa en ninguna de las variables examinadas (Tabla 1).

Tabla 1. Diferencias en quejas somáticas en función de presentar o no sintomatología depresiva y del sexo

\begin{tabular}{|c|c|c|c|c|c|c|c|c|}
\hline & & \multicolumn{2}{|c|}{$\begin{array}{l}\text { Niños con síntomas } \\
\text { depresivos } \\
(n=50)\end{array}$} & \multicolumn{2}{|c|}{$\begin{array}{l}\text { Niños sin síntomas } \\
\text { depresivos } \\
(n=616)\end{array}$} & \multicolumn{2}{|l|}{$\mathrm{F}$} & \multirow[b]{2}{*}{$S \times S D$} \\
\hline & & M & D.T & M & D.T & $\mathrm{S}$ & SD & \\
\hline \multirow[t]{3}{*}{ Síntomas pseudoneurológicos } & Niños & 8,33 & 7,84 & 4,06 & 4,40 & $4,77^{*}$ & $66,22^{* * *}$ & 4,15 \\
\hline & Niñas & 11,24 & 5,33 & 4,20 & 4,60 & & & \\
\hline & Total & 10,02 & 6,60 & 4,12 & 4,46 & & & \\
\hline \multirow[t]{3}{*}{ Síntomas gastrointestinales } & Niños & 3,00 & 3,20 & 1,64 & 2,54 & $12,06 * * *$ & $30,90 * * *$ & 5,56 \\
\hline & Niñas & 5,40 & 4,14 & 2,11 & 2,80 & & & \\
\hline & Total & 4,40 & 3,92 & 1,20 & 2,70 & & & \\
\hline \multirow[t]{3}{*}{ Dolor } & Niños & 3,95 & 3,37 & 1,84 & 2,61 & 0,33 & $38,62 * * *$ & 0,44 \\
\hline & Niñas & 4,28 & 3,70 & 1,65 & 2,28 & & & \\
\hline & Total & 4,14 & 3,53 & 1,75 & 2,45 & & & \\
\hline
\end{tabular}

Nota. $* p<.05, * * * p<.001, \mathrm{~S}=$ sexo, $\mathrm{SD}=$ sintomatología depresiva 
Diferencias en la manifestación de cada síntoma en función de la sintomatología depresiva

Se han hallado diferencias significativas en función de presentar o no sintomatología depresiva en el porcentaje de niños que manifiestan muchas o muchísimas veces (puntuaciones iguales o mayores a 3) en la mayoría de los síntomas somáticos que examina el CSI. Los niños con sintomatología depresiva presentan con mayor frecuencia la mayoría de las quejas somáticas analizadas, exceptuando dia- rrea, dolores de estómago, latidos del corazón muy rápidos, pérdida de voz, visión borrosa, ceguera, desmayos, amnesia, problemas al caminar, parálisis muscular, mala digestión y dolor de brazos o piernas, en los que no se han hallado diferencias. Los síntomas somáticos más frecuentes en los niños con sintomatología depresiva son el dolor de rodillas, codos o articulaciones (36\%), el dolor de cabeza (20\%) y la falta de energía o cansancio (20\%) (Tabla 2).

Tabla 2. Frecuencia (y porcentaje) de niños que manifiestan tener muchas o muchísimas veces cada síntoma somático

Dolor de cabeza

Mareos o sensación de desmayarse

Dolor en el pecho o en el corazón

Falta de energía o cansancio

Dolor en la parte baja de la espalda

Dolor de músculos

Falta de respiración

(cuando no estás haciendo ejercicio)

Calor o demasiado frío sin ninguna razón

Adormecimiento, entumecimiento o picazón en alguna parte del cuerpo

Tener un nudo en la garganta

Debilidad en el cuerpo

Sentir pesados los brazos o las piernas

Náuseas o molestias en el estómago

Estreñimiento al ir al baño

Diarrea al ir al baño

Dolores de estómago o abdomen

Te late el corazón muy rápido

(cuando no estás haciendo ejercicio)

Dificultad para tragar

Pérdida de voz

No poder oír (sordera)

Doble visión (incluso con gafas)

Visión borrosa (incluso con gafas)

No poder ver (ceguera)

Desmayos o pérdida de conocimiento

Pérdida de memoria o amnesia

Convulsiones (temblores rápidos en el cuerpo que no puedes controlar)

Problemas al caminar

Parálisis o debilidad muscular

Dificultad para orinar

Vomitar

Gases o inflamación en el estómago

Mala digestión (comer te hace sentir enfermo)

Dolor de rodillas, codos o articulaciones

Dolor de brazos o piernas

Dolor cuando orinas

Nota. $* p<.05,{ }^{* *} p<.01,{ }^{* * *} p<.05$

$\begin{array}{lc}\text { Niños con síntomas } & \text { Niños sin síntomas de- } \\ \text { depresivos }(n=50) & \text { presivos }(n=616)\end{array}$

\begin{tabular}{|c|c|c|}
\hline $\mathrm{n}(\%)$ & n (\%) & $x^{2}$ \\
\hline $10(20)$ & $30(4.9)$ & $17.326^{* * *}$ \\
\hline $9(18)$ & $12(2)$ & $36.780 * * *$ \\
\hline $4(8)$ & $10(1.6)$ & $8.503^{* *}$ \\
\hline $10(20)$ & $20(3.2)$ & $28.236^{* * *}$ \\
\hline $5(10)$ & $17(2.8)$ & $7.219 * *$ \\
\hline $5(10)$ & $18(2.9)$ & $6.366 *$ \\
\hline $5(10)$ & $16(2.6)$ & $7.649^{* *}$ \\
\hline $5(10)$ & $23(3.7)$ & $4.042^{*}$ \\
\hline $5(10)$ & $14(2.3)$ & $9.212^{* *}$ \\
\hline $4(8)$ & $5(0.8)$ & $16.914^{* * *}$ \\
\hline $5(10)$ & $5(0.8)$ & $24.922^{* * *}$ \\
\hline $5(10)$ & $4(0.6)$ & $28.689^{* * *}$ \\
\hline $8(16)$ & $12(2)$ & $29.500^{* * *}$ \\
\hline $3(6)$ & $6(1)$ & $8.204^{* *}$ \\
\hline $1(2)$ & $2(0.3)$ & 2.784 \\
\hline $2(4)$ & $10(1.6)$ & 1.307 \\
\hline $4(8)$ & $22(3.6)$ & 2.121 \\
\hline $2(4)$ & $4(0.6)$ & $5.445^{*}$ \\
\hline $1(2)$ & $8(1.3)$ & 0.149 \\
\hline $3(6)$ & $4(0.6)$ & $12.003^{* *}$ \\
\hline $3(6)$ & $9(1.5)$ & $5.140^{*}$ \\
\hline $2(4)$ & $7(1.1)$ & 2.603 \\
\hline $1(2)$ & $2(0.3)$ & 2.784 \\
\hline $0(0)$ & $0(0)$ & - \\
\hline $0(0)$ & $5(0.8)$ & 0.429 \\
\hline $2(4)$ & $4(0.6)$ & $5.433^{*}$ \\
\hline $1(2)$ & $6(1)$ & 0.407 \\
\hline $0(0)$ & $4(0.6)$ & 0.343 \\
\hline $3(6)$ & $0(0)$ & $35.421^{* * *}$ \\
\hline $5(10)$ & $4(0.6)$ & $28.741^{* * *}$ \\
\hline $4(8)$ & $14(2.3)$ & $5.249 *$ \\
\hline $1(2)$ & $3(0.5)$ & 1.640 \\
\hline $18(36)$ & $5(0.8)$ & $6.366^{*}$ \\
\hline $4(8)$ & $19(3.1)$ & 3.006 \\
\hline $2(4)$ & $2(0.3)$ & $9.880^{* *}$ \\
\hline
\end{tabular}

Summa Psicológica UST (CC - BY - 3.0) ISSN: 0718-0446 / ISSNe: 0719-448x http://summapsicologica.cl/ 


\section{Discusión}

El objetivo del presente estudio era examinar las quejas somáticas en niños españoles de 8 a 12 años en función de que manifiesten o no sintomatología depresiva, teniendo en cuenta su sexo y edad. Los resultados obtenidos indican que existen diferencias en la presencia de quejas somáticas en los niños con síntomas depresivos frente a aquéllos que no muestran dicha sintomatología. Además, las niñas presentaban en general más quejas somáticas que los niños.

En base a la literatura previa (Del Barrio, 2007), se esperaba que la proporción de niños españoles con síntomas depresivos estuviera comprendida entre un 4.2 y un $10 \%$. El presente estudio, en el que se hallado un porcentaje del $8 \%$ de niños con sintomatología depresiva, confirma por tanto con datos más recientes la prevalencia encontrada en estudios anteriores. La ausencia de diferencias significativas respecto al sexo apoya de igual modo las conclusiones de otros trabajos previos que señalan que es a partir de la adolescencia cuando la sintomatología depresiva difiere según el sexo (Abad et al., 2002; Bernaras et al., 2013).

Respecto a las diferencias en las quejas somáticas en función de manifestar o no síntomas depresivos, los resultados confirman la hipótesis de que las quejas somáticas son más frecuentes en los niños con sintomatología depresiva que en los que no presentan síntomas de depresión. Las diferencias se han observado en todos los síntomas examinados (pseudoneurológicos, gastrointestinales y de dolor), hallándose un tamaño del efecto medio. De este modo, los resultados del presente estudio confirman los hallazgos de trabajos previos que encuentran una relación positiva entre la depresión y la manifestación de quejas somáticas en la etapa escolar. La literatura disponible informa de una probabilidad mayor de manifestar dolor abdominal recurrente en los niños y adolescentes con diagnóstico de ansiedad o depresión (Campo et al., 2004). Los dolores musculares se han relacionado con depresión tanto en niñas como en niños de 9 a 16 años (Egger, Costello, Erkanli \& Angold, 1999), y también el dolor abdominal se ha asociado con la sintomatología depresiva, teniendo los adolescentes con dolor abdominal frecuente un riesgo de entre un 16 y un $45 \%$ de presentar sintomatología depresiva (Youssef, Atienza, Langseder \& Strauss, 2008). El dolor de cabeza (Anttila et al., 2004) y los trastornos gastrointestinales funcionales (Yacob et al., 2013) también se han relacionado con frecuencia con la depresión en niños y adolescentes. Además, las quejas somáticas no solo se han asociado con la sintomatología depresiva en la etapa escolar, sino también en la adultez, de modo que manifestar con frecuencia quejas somáticas en la infancia predice la presencia de problemas emocionales en la etapa adulta, en concreto depresión y ansiedad generalizada (Shanahan et al., 2015).

De acuerdo a estudios anteriores que han mostrado una mayor predisposición en las niñas a manifestar quejas somáticas (Jellesma, Rieffe \& Terwogt, 2008; Serra et al., 2013), los resultados del presente trabajo confirman que existe una mayor presencia de quejas de tipo pseudoneurológico y gastrointestinales en la población infantil femenina que en la masculina. En concreto, hallazgos previos informan de una mayor prevalencia de mareos y dolor de cabeza en niñas que en niños de 10 a 12 años (Ordóñez et al., 2015). Estudios longitudinales internacionales también han confirmado niveles más altos de quejas somáticas en adolescentes mujeres que en varones (Ruchkin \& Schwab-Stone, 2014). Al examinar el porcentaje de participantes de nuestro estudio que manifiestan cada queja somática muchas o muchísimas veces, se observa que los que tienen síntomas depresivos presentan la mayoría de quejas somáticas con mayor frecuencia que los niños sin sintomatología depresiva. Las quejas somáticas más prevalentes en los niños con síntomas depresivos son el dolor de articulaciones (36\%), el dolor de cabeza (20\%), la falta de energía o cansancio (20\%), los mareos (18\%) y las náuseas o dolor de estómago (16\%). En los niños sin sintomatología depresiva el dolor de cabeza (4.9\%) es la queja somática más frecuente pero su prevalencia es mucho menor que en los niños con síntomas depresivos. Los resultados de nuestro trabajo confirman en este sentido los hallazgos de estudios anteriores que concluyen que el dolor de cabeza y el dolor de estómago son las quejas somáticas más frecuentes en la población infantil general en estas edades (Ordóñez et al., 2015; Serra et al., 2013).

El presente estudio presenta ciertas limitaciones y fortalezas. Su principal fortaleza es que aporta nuevas evidencias sobre la relación entre la depresión infantil y las quejas somáticas. En comparación con los trabajos previos llevados a cabo, se incluye una amplia variedad de quejas somáticas no examinadas previamente en los estudios con muestras españolas, como las náuseas, los problemas para respirar o la dificultad para tragar. Como principal limitación destacamos que se ha examinado sintomatología depresiva pero no niños con un diagnóstico de depresión puesto que la información se ha recogido a través de autoinformes y no de una entrevista diagnóstico. Al tratarse de un estudio transversal, los resultados deben tomarse con cautela, siendo recomendable llevar a cabo en el futuro investigaciones longitudinales que proporcionen datos más certeros sobre la relación entre la sintomatología depresiva y las quejas somáticas. Las implicaciones clínicas del estudio son claras. Dada la frecuencia de las quejas somáticas en los niños con síntomas depresivos, es importante detectarlas con el fin de que se atiendan debidamente en las intervenciones de la depresión infantil. Del mismo modo, la detección de síntomas somáticos no explicados por una enfermedad física en las consultas pediátricas posibilitaría la derivación e intervención en los menores atendiendo a esas manifestaciones somáticas como síntomas de problemas emocionales. 


\section{Referencias}

Abad, J., Forns, M. \& Gómez, J. (2002). Emotional and behavioral problems as measured by YSR: Gender and age differences in Spanish adolescents. European Journal of Psychological Assessment, 18, 149-157.

Angold, A., Erkanli, A., Silberg, J., Eaves, L. \& Costello, E. J. (2002). Depression scale scores in 8-17-year-olds: Effects of age and gender. Journal of Child Psychology and Psychiatry, 43(8), 1052-63.

Anttila, P., Sourander, A., Metsahonkala, L., Aromaa, M., Helenius, H. \& Sillanpaa, M. (2004). Psychiatric symptoms in children with primary headache. Journal of the American Academy of Child and Adolescent Psychiatry, 43, 412-419.

Baji, I., Lopez-Duran, N. L., Kovacs, M., George, C. J., Mayer, L., Kapornai, K., ... Vetró, A. K. (2009). Age and sex analyses of somatic complaints and symptom presentation of childhood depression in a Hungarian clinical sample. The Journal of Clinical Psychiatry, $70,1467-1472$.

Barros-Vanegas, O. B., Mercado-Osorio, S. P. \& Solano-Lamadrid, D. (2013). Prevalencia de sintomatología de depresión infantil en niños y niñas de $4^{\circ} \mathrm{A} 6^{\circ}$ grado pertenecientes a dos colegios oficiales de la ciudad de Barranquilla (Tesis doctoral, Fundación Universitaria Los Libertadores). Recuperada de http:// www.redalyc.org/pdf/1390/139031679015.pdf

Beltrán, G. \& Mora, C. (2011). Prevalencia de la depresión infantil en un grupo de estudiantes entre los 9 y 12 años de un colegio del municipio de Cajica. Recuperado de http://intellectum.unisabana.edu.co:8080/jspui/bitstream/10818/1339/1/Giormary\%20Beltr\%C3\%A1n\%20Osorio.pdf

Bernaras, E., Jaureguizar, J., Soroa, M., Ibabe, I. \& de las Cuevas, C. (2013). Evaluación de la sintomatología depresiva en el contexto escolar y variables asociadas. Anales de psicología, 29(1), 131-140.

Campo, J. V., Bridge, J., Ehmann, M., Altman, S., Lucas, A., Birmaher, B., ... Brent, D. A. (2004). Recurrent Abdominal Pain, Anxiety, and Depression in Primary Care. Pediatrics, 113(4), 817-824.

Cicchetti, D. \& Cohen, D. (Eds.) (2006). Developmental psychopathology: Risk, disorder, and adaptation (2a ed., Vol. 3). New York: Wiley.

Del Barrio, V. (2007). El niño deprimido. Barcelona: Ariel.

Del Barrio, V. (2008). Tratando...depresión infantil. Madrid, España: Pirámide.

Del Barrio, V. \& Carrasco, M.A. (2004). CDI. Inventario de depresión infantil. Madrid: TEA Ediciones S.A.

Domènech-Llaberia, E., \& Polaino-Lorente, A. (1990). La escala ESDM como instrumento adicional en el diagnóstico de la depresión infantil. Revista de Psiquiatría de la Facultad de Medicina de Barcelona, 17(3), 105-113.

Egger, H. L., Costello, E. J., Erkanli, A. \& Angold, A. (1999). Somatic complaints and psychopathology in children and adolescents: stomach aches, musculoskeletal pains and headaches. Journal of American Academy of Child and Adolescent Psychiatry, 38(7), 852-60.

Ezpeleta, L., Osa, N., Gratacos, L. L. \& Pons, D. (1992). Depresión y factores asociados en niños con puntuaciones extremas del CDI. Revista de Psiquiatría Infan- to-juvenil, 1, 39-49.

García-Vera, M.P. \& Sanz, J. (2012). Depresión. En M. I. Comeche \& M. A. Vallejo (Eds.), Manual de terapia de conducta en la infancia (pp. 197-240). Madrid: Dykinson.

Grupo de Trabajo de la Guía de Práctica Clínica sobre la Depresión Mayor en la Infancia y en la Adolescencia. (2009). Guía de Práctica Clínica sobre la Depresión Mayor en la Infancia y en la Adolescencia. Plan de Calidad para el Sistema Nacional de Salud del Ministerio de Sanidad y Política Social. Axencia de Avaliación de Tecnoloxías Sanitarias de Galicia (avalia-t). Guías de Práctica Clínica en el SNS: avalia-t No 2007/09.

Guillén, E., Gordillo, M. J., Ruiz, I., Gordillo, M. D. \& Gordillo, T. (2013). ¿Depresión o evolución?: Revisión histórica y fenomenológica del concepto aplicado a la infancia y adolescencia. International Journal of Developmental and Educational Psychology: INFAD. Revista de Psicología, 2(1), 499-506.

Jellesma, F., Rieffe, C. \& Terwogt, M. M. (2008). My peers, my friend, and I: Peer Interactions and somatic complaints in boys and girls. Social Science \& Medicine, 66, 2195-2205.

Kovacs, M. (1992). The Children's Depression Inventory (CDI). Toronto, Ontario, Canada, Multi-Health Systems.

Kranzler, A., Young, J. F., Hankin, B., Abela, J. R., Elias, M. J. \& Selby, E. A. (2016). Emotional awareness: A transdiagnostic predictor of depression and anxiety for children and adolescents. Journal of Clinical Child and Adolescent Psychology, 45(3), 262-269. doi:10.1 080/15374416.2014.987379

López, C., Alcántara, M. V., Fernández, V., Castro, M. \& López, J. A. (2010). Características y prevalencia de los problemas de ansiedad, depresión y quejas somáticas en una muestra clínica infantil de 8 a 12 años, mediante el CBCL (Child Behavior Checklist). Anales de psicología, 26(2), 325-334.

Ordóñez, A., Maganto, C. \& González, R. (2015). Quejas somáticas, conciencia emocional e inadaptación en población escolar. Anales de Pediatría, 82(5), 308315. doi: 10.1016/j.anpedi.2014.03.020

Orgilés, M. \& Espada, J. P. (2014). Spanish Version of the Children's Somatization Inventory: Factorial Structure and Psychometric Properties in a Community Sample. International Journal of Behavioral Medicine, 21(3), 556-560.

Rieffe, C. \& De Rooij, M. (2012). The longitudinal relationship between emotion awareness and internalizing symptoms during late childhood. European Child and Adolescent Psychiatry, doi: 10.1007/s00787012-0267-8

Rieffe, C., Meerum Terwogt, M. \& Jellesma, F. C. (2008). Emotional competence and health in children. En J. Denollet, I. Nyklicek \& A. Vingerhoets (Eds.), Emotion Regulation: Conceptual and Clinical Issues (pp. 184-201). New York: Springer.

Rieffe, C., Oosterveld, P., Terwogt, M. M., Novin, S., Nasiri, H. \& Latifian, M. (2010). Relationship between alexithymia, mood and internalizing symptoms in children and young adolescents: Evidence from an Iranian sample. Personality and Individual Differences, 48, 425-430.

Ruchkin, V. \& Schwab-Stone, M. (2014). A longitudinal study 
of somatic complaints in urban adolescents: the role of internalizing psychopathology and somatic anxiety. Journal of Youth \& Adolescence, 43(5), 834-845.

Serra, R., Claustre, M. \& Bonillo, A. (2013). Síntomas somáticos funcionales en una muestra española: psicopatología y estilos educativos. Anales de Pediatría, 79, 101-107.

Shanahan, L., Zucker, N., Copeland, W. E., Bondy, C. L., Egger, H. L. \& Costello, E. J. (2015). Childhood somatic complaints predict generalized anxiety and depressive disorders during young adulthood in a community sample. Psychological medicine, 45(8), 1721-1730.

Walker, L. S., Beck, J. E., Garber, J. \& Lambert, W. (2009). Children's Somatization Inventory: psychometric properties of the revised form (CSI-24). Journal of Pediatric Psychology, 34(4), 430-440.

Yacob, D., Di Lorenzo, C., Bridge, J. A., Rosenstein, P. F., Onorato, M., Bravender, T. \& Campo, J. V. (2013). Prevalence of pain-predominant functional gastrointestinal disorders and somatic symptoms in patients with anxiety or depressive disorders. The Journal of Pediatrics, 163, 767-770.

Youssef, N.N., Atienza, K., Langseder, A.L. \& Strauss, R.S. (2008). Chronic abdominal pain and depressive symptoms: Analysis of the National Longitudinal Study of Adolescent Health. Clinical Gastroenterology Hepatology, 6, 329-332.

Zolog, T., Claustre, M., Bonillo, A., Canals-Sans, J., Hernández-Martínez, C., Romero-Acosta, K. \& Domenech-Llaberia, E. (2011). Somatic complaints and symptoms of anxiety and depression in a school-based sample of preadolescents and early adolescents. Functional impairment and implications for treatment. Journal of Evidence-Based Psychotherapies, 11, 191-208. 